\title{
Clinical Aspects and Conservative Dental Management of a Patient with Fibrodysplasia Ossificans Progressiva
}

\author{
${ }^{1}$ Francisco Artur Forte Oliveira, ${ }^{2}$ Clarissa Pessoa Fernandes, ${ }^{3}$ Kenia Samara Barbosa Araujo \\ ${ }^{4}$ Ana Paula Negreiros Nunes Alves, ${ }^{5}$ Fabrício Bitu Sousa, ${ }^{6}$ Mário Rogério Lima Mota
}

\section{ABSTRACT}

Aim: To present the clinical findings of a patient with Fibrodysplasia Ossificans Progressiva (FOP), highlighting peculiarities of dental treatment in patients with this condition.

Background: FOP is a rare genetic disease characterized by skeletal malformations and ectopic ossifications in skeletal muscles, tendons, ligaments and aponeurosis. Exacerbation of these ossifications can be caused by dental treatment, resulting in disease progression.

Case description: A 26-year-old male patient with a diagnosis of FOP was referred to our service for dental treatment. The patient presented decreased mobility in peripheral joints (knees and elbows), postural disability (ankylosis of the vertebral column), lateral deviation and shortness of the hallux, as well as heterotopic ossifications on the hands and back. The implementation of conservative dental procedures, such as oral hygiene instructions and recurrent topical fluoride applications, were performed in addition to endodontic and restorative treatments. Brief dental appointments were conducted without using regional anesthesia or dental dam clamps. The dental chair was positioned at $45^{\circ}$ to provide more comfort and to avoid exacerbating the disease. The patient has now completed 6 months of follow-up and is free of heterotopic ossifications resulting from dental treatment.

Conclusion: The dental treatment modifications implemented for the present case were sufficient to establish good oral health and to prevent the formation of heterotopic ossifications in the maxillofacial region.

Clinical significance: FOP is a rare disease dentists must familiarize themselves with to provide adequate, personalized treatment, which minimizes traumas that may exacerbate the disease.

Keywords: Fibrodysplasia ossificans progressiva, Clinical characteristics, Dentistry, Dental care, Restorative treatment, Endodontic treatment, Preventive treatment, Case report.

\footnotetext{
1,2Doctorate Student, ${ }^{3}$ Undergraduate Dental Student,

${ }^{4-6}$ Associate Professor

1-3,5,6 Department of Stomatology and Oral Pathology Federal University of Ceara, Ceara, Brazil

${ }^{4}$ Department of Oral Pathology, Federal University of Ceara Ceara, Brazil
}

Corresponding Author: Francisco Artur Forte Oliveira Department of Stomatology and Oral Pathology, Federal University of Ceara, Ceara, Brazil, e-mail: arturforte@ymail.com
How to cite this article: Oliveira FAF, Fernandes $C P$, Araujo KSB, Alves APNN, Sousa FB, Mota MRL. Clinical Aspects and Conservative Dental Management of a Patient with Fibrodysplasia Ossificans Progressiva. J Contemp Dent Pract 2014;15(1):122-126.

\section{Source of support: Nil}

Conflict of interest: None declared

\section{INTRODUCTION}

Fibrodysplasia ossificans progressiva (FOP), also known as Myositis Ossificans Progressiva, is a rare genetic disease (autosomic dominant) that affects approximately one in two million people. This disease is characterized by skeletal malformations, especially of the toes, and by heterotopic progressive ossifications in skeletal muscles, tendons, ligaments and aponeuroses. $^{1,2}$

The first heterotopic ossifications usually manifest during the first decade of life, mainly affecting tissue surrounding the hipbones, vertebral column and joints. ${ }^{3}$ The maxillofacial region can also be affected, and when the masticatory muscles are involved (the masseter, medial and lateral pterygoids, temporalis and buccinators), as well as the temporomandibular joint (TMJ), constraints on opening the mouth and subsequent eating difficulty can occur in addition to difficulty in maintaining oral hygiene. ${ }^{4}$

Performing dental procedures may be necessary because FOP patients may have a high risk of developing caries and periodontal disease. In these patients, dental treatment can be complicated due to TMJ ankylosis (which imposes strictures on opening the mouth), ankylosis of the joints of the arms and legs and ankylosis of the vertebral column, accounting for postural changes. ${ }^{5}$ Dental treatment can exacerbate ossifications in the maxillofacial region; therefore, measures to minimize ossifications, including avoiding regional anesthesia and surgical procedures, must be implemented whenever possible. ${ }^{2}$

In this case report, nonsurgical dental treatment of a 26-year-old patient diagnosed with FOP will be discussed, with particular emphasis on the peculiarities dentists must consider to provide safe and personalized treatment to these patients. 


\section{CASE DESCRIPTION}

A 26-year-old male patient, diagnosed with FOP at the age of 18, was referred to the School of Dentistry of the Federal University of Ceara (UFC), Fortaleza, Brazil, for dental treatment. The patient was not taking any drugs for treatment and was being monitored by orthopedics, cardiology and pulmonology. The patient presented clinical characteristics of the disease such as decreased mobility of peripheral joints (knees and elbows), vertebral column involvement, accounting for postural deficiency (Fig. 1), lateral deviation and shortness of the hallux (Fig. 2) and heterotopic ossifications on the hands and back (Figs 3 and 4).

Assessment of the maxillomandibular region revealed satisfactory ability to open the mouth and also revealed mandibular hypoplasia, caries, and periodontal disease (visible dental plaque and dental calculus). Radiographic evaluation was made only through viewing periapical X-rays because the patient's postural disability precluded panoramic X-rays.

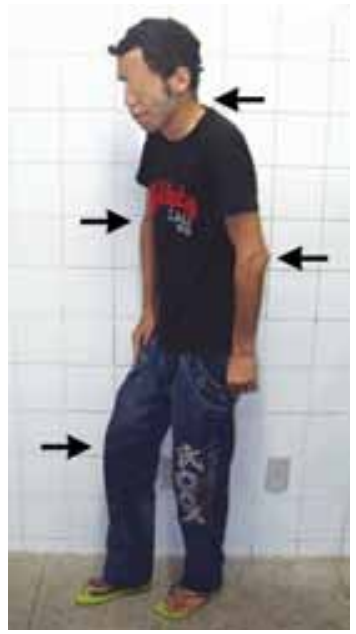

Fig. 1: General aspect of the patient showing postural deficiency due to ankylosis of peripheral joints (knees and elbows) and vertebral column

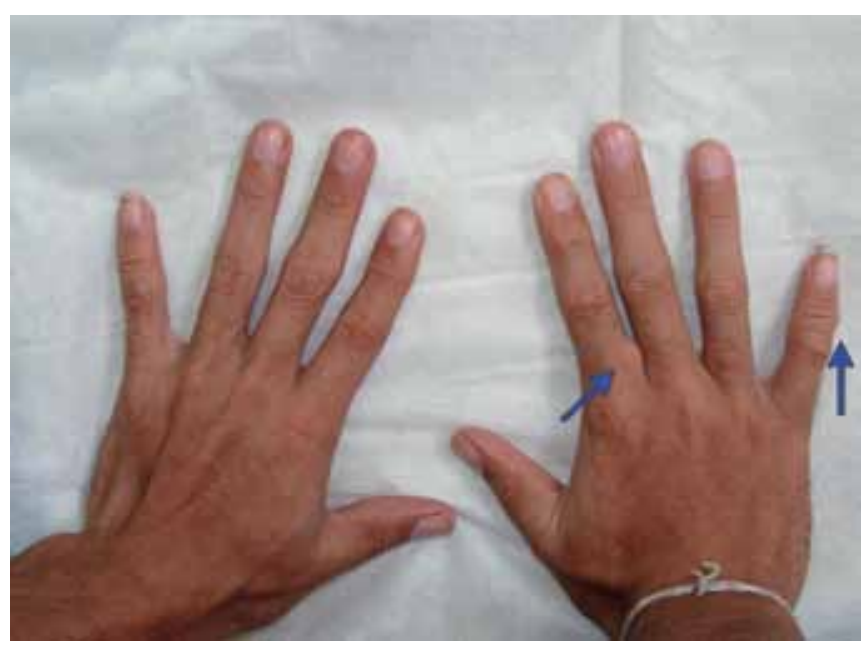

Fig. 3: Heterotopic ossifications on the hands
Periapical X-rays showed a previous endodontic treatment of the left maxillary first molar and demonstrated the necessity of endodontic treatment for the left maxillary second premolar. Restorative dental procedures were also indicated.

Dental management included oral hygiene instructions, dental plaque control, recurrent topical fluoride applications and periodontal treatment (supragingival scaling) in addition to endodontic and restorative treatments (Figs 5A and B). Only one procedure was performed at each dental appointment to ensure that appointments remained brief. Regional anesthesia or dental dam clamps were not used (Fig. 6). The dental chair was positioned at $45^{\circ}$, and the patient's neck was kept straight to avoid hyperextension of the neck and provide greater comfort and safety.

The patient has now completed 6 months of follow-up and is free of heterotopic ossifications resulting from dental treatment. The patient's ability to open his mouth has remained the same since his initial appointment.

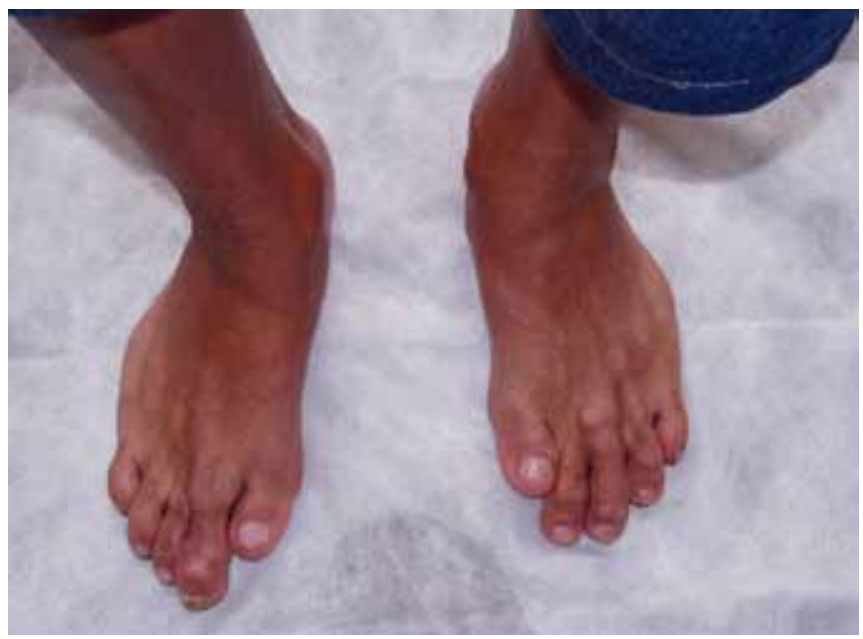

Fig. 2: Skeletal malformation of the feet (shortness and lateral deviation of the great toes)

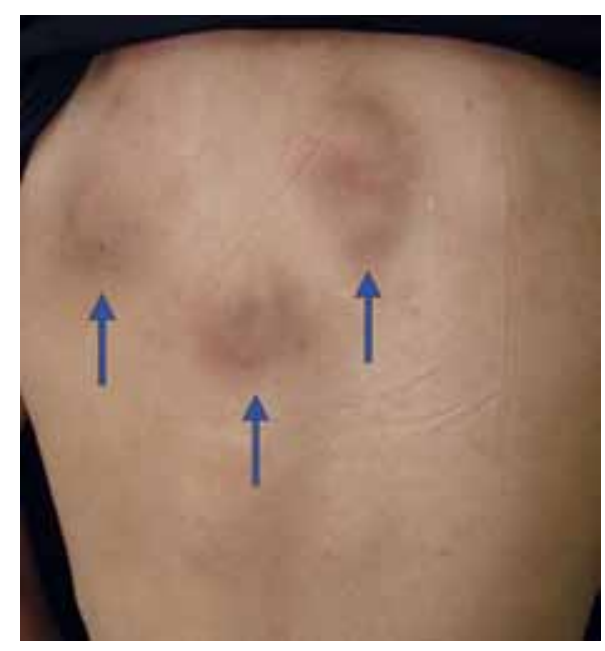

Fig. 4: Heterotopic ossifications on the back 


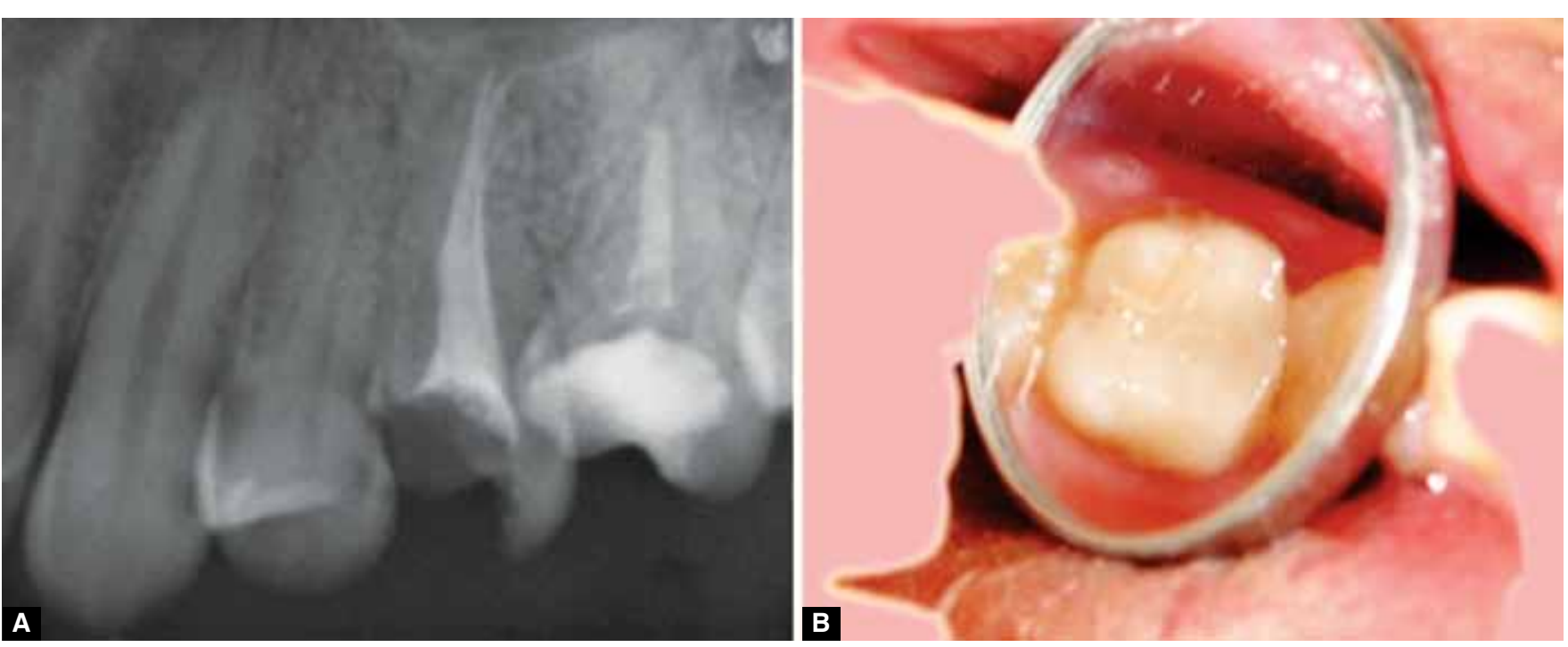

Figs 5A and B: Endodontic treatment of the left maxillary second premolar, (B) clinical aspect of the restorative treatment of the left maxillary first molar

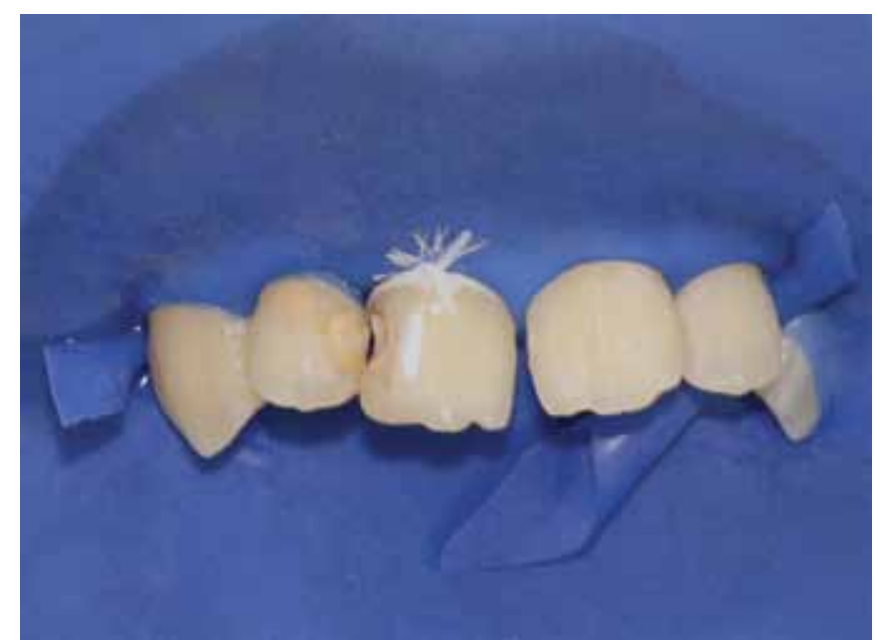

Fig. 6: Modified dental dam with floss ligature (without dental dam clamp)

\section{DISCUSSION}

FOP represents a genetic condition in which skeletal muscles, ligaments, tendons and joints in the arms, legs, back and vertebral column display heterotopic ossifications preceded by sporadic soft-tissue swellings (flare-ups) ${ }^{6}$ the maxillofacial region can also be involved. Calcifications of the TMJ and masticatory muscles can occur spontaneously during the natural clinical course of this disease or can be induced and exacerbated by dental treatment. ${ }^{7}$

The first heterotopic ossifications in FOP appear within the first decade of life (3 to 6 years old). These ossifications usually advance from axial to appendicular areas and from cranial to caudal anatomic locations. The neck, shoulders, vertebral column and elbows are initially involved. ${ }^{8}$ In the present case, although the patient presented clinical signs of the disease since birth (malformations of the great toes), the FOP diagnosis was made 3 years after the appearance of the first heterotopic ossifications when the patient was 15 years old. The ossifications progressively involved his hands, elbows, feet, knees, back and vertebral column with fusion of the ligaments between $\mathrm{C} 2$ and $\mathrm{C} 7$. This phenomenon resulted in scoliosis, reduction of the neck's mobility, movement impairment and difficulties in performing daily hygiene like brushing his teeth. The patient also presented slight mandibular hypoplasia, which is a normal maxillofacial finding in patients with FOP, as described by Roberts et al. ${ }^{2}$

Patients with FOP do not have more dental problems than do the general population, except when mandible ankylosis occurs or when heterotopic ossifications of the hands and elbows prevent adequate toothbrushing. Although this patient did not present with limitations to opening his mouth (TMJ ankylosis), he still had difficulties performing oral hygiene, due to heterotopic ossifications of his elbows and hands. To facilitate oral hygiene, an electric toothbrush was indicated, as it has proven efficacious in removing dental plaque for patients with motor disorders. ${ }^{9}$ Oral hygiene instructions were given to the patient's mother to ensure that she could help to improve his oral health.

Because FOP patients can experience progressive limitations in opening their mouths, preventive dental treatment becomes essential, especially during infancy. Periodontal treatment, the use of high-dose fluoride toothpaste and recurrent topical fluoride applications help to prevent the development of oral diseases. Antimicrobial (chlorhexidine) and fluoride rinses should also be used, especially in cases where it is not possible to reach the lingual and palatal surfaces by traditional brushing methods. ${ }^{5,10}$

When preventive treatment is not performed or proves unsuccessful, invasive oral interventions become necessary. 
During any dental procedures, the FOP patient must remain as confortable as possible. Keeping dental appointments brief and positioning the dental chair at $45^{\circ}$ help prevent overextending the neck and mandible, maintain the patient's head alignment with the body, and minimize additional traumas. ${ }^{11}$ Hyperextension of the jaws, mandibular anesthetic blocks and surgical oral procedures are associated with abnormal bone deposition; therefore, measures to avoid these conditions must be implemented whenever possible. Although local anesthesia options in FOP patients are limited, intramuscular injections, including mandibular blocks, must be avoided because they can initiate heterotopic ossifications of the facial muscles and can rapidly induce TMJ ankylosis. ${ }^{5}$

An alternative to regional anesthesia is infiltrative injections limited to the mucosa, which, with caution, can be used for maxillary teeth, including posterior teeth. For the posterior mandibular teeth, intrapulpal or intraligamental injections can be used to avoid regional anesthesia. However, this alternative is not always possible, due to difficulty in opening the mouths of patients with severe limitations.In cases in which general anesthesia becomes necessary, fiber optic nasal intubation is recommended because oral intubation can cause additional trauma to the mandible.,

For patients who have TMJ ankylosis and experience challenges in opening their mouths, preparing teeth and removing decay becomes difficult, sometimes necessitating a slow dental drill to access the tooth's buccal surface. Using fluoride-releasing filling material is recommended to help to prevent further decay. ${ }^{5,11}$ In the present case, because the patient could open his mouth satisfactorily, restorative treatment was performed by the conventional technique with a few minimal modifications, such as using a dental dam without clamps (alternatively, floss ligatures were used to hold the rubber dam in the gingival crevice).

In patients with severe mouth-opening limitations, surgical intervention (dental extractions) becomes the only option for endodontically compromised teeth due to difficulties in performing endodontic treatment. This restriction explains the paucity of studies in the literature that describe endodontic treatment in FOP patients. In cases calling for surgical intervention, dental extractions may be difficult to perform. Placing forceps on top of the tooth is sometimes impossible, necessitating using the buccal approach: sectioning the tooth and forming a window on the buccal side is the technique usually used in these cases. ${ }^{5}$ Because the patient in the present case did not present TMJ ankylosis, an endodontic procedure was included in the treatment plan and was performed under intrapulpal anesthesia with a dental dam employing floss ligatures.
FOP must be diagnosed early to ensure that it can be differentiated from other ossifying diseases and neoplastic processes, thereby reducing iatrogenic procedures caused by health professionals, including dentists. There is still no cure or effective proven treatment for FOP, but advances in genetics and technology are promising for FOP treatment.

\section{CONCLUSION}

The management of the patient and the modifications implemented during dental treatment were adequate to establish sound oral health and to prevent traumas that could lead to heterotopic ossifications of the masticatory muscles and TMJ. In addition to preventing impairment in opening the mouth, these procedures helped maintain the patient's ability to chew, speak and swallow without complications.

\section{CLINICAL SIGNIFICANCE}

It is important that dentists understand the peculiarities and characteristics of FOP such that they can provide adequate and personalized dental treatment, minimizing traumas and iatrogenic procedures that might exacerbate the progression of FOP.

\section{ACKNOWLEDGMENTS}

The authors are grateful to FUNCAP (Fundação Cearense de Apoio ao Desenvolvimento Científico e Tecnológico) and to CAPES (Coordenação de Aperfeiçoamento de Pessoal de Nível Superior) for providing financial support through scholarships for postgraduate students.

\section{REFERENCES}

1. Trigui M, Ayadi K, Zribi M, Triki Z, Keskes H. Fibrodysplasiaossificans progressiva: diagnosis and surgical management. Acta Orthop Belg 2011;77:139-144.

2. Roberts T, Stephen L, Scott C, Urban M, Sudi S, Beighton P. Fibrodysplasia ossificans progressiva (FOP) in South Africa: dental implications in 5 cases. Oral Surg Oral Med Oral Pathol Oral Radiol Endod 2011;112:11-18.

3. Vashisht R, Prosser D. Anesthesia in a child with fibrodysplasiaossificans progressiva. Paediatr Anaesth 2006;16:684-688.

4. Mori Y, Susami T, Haga N, Tamura K, Kanno Y, Saijo H, Takato T. Extraction of 6 molars under general anesthesia in patient with fibrodysplasia ossificans progressiva. J Oral Maxillofac Surg 2011;69:1905-1910.

5. Nussbaum BL, Grunwald Z, Kaplan FS. Oral and dental health care and anesthesia for persons with fibrodysplasiaossificans progressiva. Clinical Reviews in Bone and Mineral Metabolism 2005;3:239-242.

6. Pignolo RJ, Shore EM, Kaplan FS. Fibrodysplasiaossificans progressiva: clinical and genetic aspects. Orphanet J Rare Dis 2011;6:80. 
7. Pignolo RJ, Suda RK, Kaplan FS. The fibrodysplasiaossificans progressiva lesion. Clinical Reviews in Bone and Mineral Metabolism 2005;3:195-200.

8. Kaplan FS, Glaser DL. Thoracic insufficiency syndrome in patients with fibrodysplasia ossificans progressiva. Clinical Reviews in Bone and Mineral Metabolism 2005;3(3):213-216.

9. De Visschere LM, van der Putten GJ, Vanobbergen JN, Schols JM, de Baat C, Dutch Association of Nursing Home Physicians.
An oral health care guideline for institutionalised older people. Gerodontology 2011;28:307-310.

10. Nussbaum BL, O’Hara I, Kaplan FS. Fibrodysplasiaossificans progressiva: report of a case with guidelines for pediatric dental and anesthetic management. ASDC J Dent Child 1996;63:448-450.

11. Young JM, Diecidue RJ, Nussbaum BL. Oral management in a patient with Fibrodysplasia ossificans progressiva. Spec Care Dentist 2007;27:101-104. 